Check for updates

Cite this: RSC Adv., 2019, 9, 30545

Received 28th July 2019

Accepted 3rd September 2019

DOI: $10.1039 / c 9 r a 05854 k$

rsc.li/rsc-advances

\section{Identification of proteins in housefly (Musca domestica) larvae powder by LC-MS/MS and their potential medical relevance}

\author{
Mengya Jiao, (D) $\dagger^{\mathrm{b}}$ Lei Chen, (D) $\dagger^{\mathrm{c}}$ Yinru He, (D) $\dagger^{\mathrm{b}}$ Lirong Wu (D) *a \\ and Hanfang Mei iD *ab
}

\begin{abstract}
Housefly larvae $(\mathrm{HL})$ powder was used to cure wounds centuries ago for its good nutritional and pharmacological values. At present, most of the medical studies are about the crude extracts of $\mathrm{HL}$, while the specific pharmacological material basis is still unclear. We ground third-instar Musca domestica larvae into a powder, degreasing and preparing the protein extract. The protein extract was subjected to enzymatic hydrolysis, and the enzymatic hydrolysis products were identified by liquid chromatographytandem mass spectrometry (LC-MS/MS). We identified a variety of highly trusted proteins (false discovery rate is less than or equal to $1 \%$ ), including catalysis-related proteins, antioxidant proteins and antimicrobial peptides, which may be closely related to the anti-tumor, anti-bacterial, anti-oxidant and other pharmacological effects of $\mathrm{HL}$. We identified the amino acid sequences of these proteins, and further confirmed HL's protective effect on APP/PS1 transgenic Alzheimer's mice. The results of this work provide material basis for further medical research on $\mathrm{HL}$.
\end{abstract}

\section{Introduction}

Insects are a large source of potentially useful compounds for modern medicine. ${ }^{1}$ The housefly (Musca domestica) belongs to Diptera, and housefly larvae (HL) are insects with high nutritional value. HL, also known as maggot or Wuguchong, is contained in the fortieth volume of the worm department, Compendium of Materia Medica. ${ }^{2}$ HL has been approved by the U.S Food and Drug Administration (FDA) to enter the market as a medical device. ${ }^{3}$ It was reported that HL is mainly composed of protein with a total content of about $62.52 \%$. The essential amino acid content is $47.72 \%$ of total amino acids ( $E \%)$, and the ratio of essential amino acids to non-essential amino acids is 0.91 , while $E \% / T$ (constant term $=15$ ) is 3.18. ${ }^{4}$ The analyzed essential amino acid ratios are higher than that of the reference protein model proposed by the Food and Agriculture Organization-World Health Organization (FAO-WHO), indicating that HL protein is a high quality protein. ${ }^{5}$

HL has been used in medicine and as a functional food for centuries, and the biological effects of HL have been studied

${ }^{a}$ Guangdong Key Laboratory of Pharmaceutical Bioactive Substances, Guangdong Pharmaceutical University, Guangzhou Higher Education Mega Center, 280 Wai Huan Dong Lu, Guangzhou, Guangdong 510006, China. E-mail: meihf37@163.com; 30872950@qq.com; Tel: +86-20-39352192; +86-20-39352552

${ }^{b}$ Department of Biochemistry and Molecular Biology, School of Life Science and Biopharmaceuticals, Guangdong Pharmaceutical University, Guangzhou Higher Education Mega Center, Guangzhou, Guangdong 510006, China

${ }^{c}$ Department of Dental Emergency, Stomatological Hospital of Southern Medical University, Southern Medical University, Guangzhou, Guangdong 510280, China

$\dagger$ These authors contributed equally to this work. extensively. Sang et al. applied water-soluble protein enzymatic hydrolysates and an ethanol-extracted fraction from HL to antimicrobial research, and demonstrated its profound antibacterial activity. ${ }^{6}$ Chu et al. used the protein-enriched extracts of HL in anti-atherosclerosis studies, and testified their anti-atherosclerotic function in mice, which may be related to the inhibition of NF- $\kappa \mathrm{B}$ p65 expression and nuclear transfer., ${ }^{7,8}$ Zhu et al. showed that the polypeptides hydrolyzed by the neutral protease of $\mathrm{HL}$ have protective effects on $\mathrm{H}_{2} \mathrm{O}_{2}$-induced oxidative damage of HePG2 cells, and the mechanisms may have the ability to reduce intracellular ROS and increase the activities of antioxidant enzymes, such as superoxide dismutase. ${ }^{9}$ Hou et al. proved that the protein extract from the third instar larvae of the housefly possesses broad antibacterial activity against both Gram-negative bacteria and Gram-positive bacteria. ${ }^{10}$ Meanwhile, it was also proved to have anti-tumor activity in vitro, in the same study. ${ }^{10}$ However, the current medical research on HL is mostly limited to its crude extract, and the specific pharmacological material basis is unclear to date. In order to further explore the pharmacological effects and molecular mechanisms of HL, it is of great significance to clarify its chemical constituents using modern science and technology.

Mass spectrometry (MS) technology has the characteristics of high sensitivity and high precision, which can identify proteins accurately and quickly. The advent and the development of new ionization techniques, such as surface-assisted laser desorption/ionization time-of-flight mass spectrometry (SALDITOF-MS), ${ }^{11}$ matrix-assisted laser desorption ionization time-offlight mass spectrometry (MALDI-TOF-MS), ${ }^{\mathbf{1 2}, 13}$ electrospray ionization mass spectrometry (ESI-MS) ${ }^{13,14}$ and LC-MS/MS, ${ }^{15}$ 
provide convenient conditions for the accurate and rapid identification of large molecules, such as proteins.

In this study, we ground third instar HL into a powder for protein extraction, obtained its enzymatic hydrolysate, and identified the proteins from enzymatic hydrolysates using LC-MS/MS. According to the identified proteins and their functions, we infer that HL may have a protective effect on Alzheimer's disease (AD), which was preliminarily verified in this work. The results should provide reference for further research on the medicinal functions of HL.

\section{Materials and methods}

\subsection{Drugs and reagents}

Chromatography grade pure acetonitrile, methanol, and formic acid were obtained from Merck Co., Ltd. (USA). DTT, IAA, TrisHCL, urea, and ammonium bicarbonate were bought from Sigma Co., Ltd. (USA). Trypsin was from Promega Corp. Donepezil was purchased from Eisai Co., Ltd. (China). Proanthocyanidins were purchased from Tianjin Jianfeng Natural Products Research and Development Co., Ltd. (China). A $\beta$ antibody was obtained from CST Co., Ltd. (USA). Other reagents were purchased from Tianjin Baishi Chemical Industry Co., Ltd. (China) or Beyotime Biotechnology Co., Ltd. (China).

\subsection{Preparation of the HL powder}

HL was purchased from Yaodetang (China). After hatching, the larvae were raised at a controlled room temperature of $25-28{ }^{\circ} \mathrm{C}$, with a relative humidity of $60-80 \%$. We cleaned the third instar larvae with distilled water and sterilized them with $70 \%$ ethanol. Physiological saline was added in a mass-to-volume ratio of $1: 1$. HL was turned into a mixture by a grinder. To obtain the HL powder, the mixture was degreased by the Soxhlet extractor method, which means that the fat in the sample was removed by reflux extraction with a low-boiling organic solvent. ${ }^{16}$ Then, it was filtered through a 100 mesh gauze, and the filtered liquid was vacuum-dried.

\subsection{LC-MS/MS analysis}

The crude HL powder was subjected to protein extraction, enzymatic hydrolysis, LC-MS/MS detection, and protein identification by using previous experimental methods, ${ }^{17}$ which are summarized as follows. We used the acetone precipitation method to precipitate the protein of the HL powder, and then carried out the protein enzymatic hydrolysis by trypsin. The mass ratio of enzyme to protein was $1: 50$, and the protein was hydrolyzed for $16 \mathrm{~h}$ at $37{ }^{\circ} \mathrm{C}$. The enzymatic peptide samples were reconstituted with a $30 \mu \mathrm{L} 0.1 \%$ formic acid/water solution. The separation parameters of high performance liquid chromatography and the parameters used in the mass spectrometry method are shown in the tables below.
High performance liquid

chromatograph

The chromatographic column

Loading volume
Thermo Scientific Easy-nLC 1000

C18, $3 \mu \mathrm{m}, 100 \AA$, $75 \mu \mathrm{m} \times 15 \mathrm{~cm}$ $1.5 \mu \mathrm{L}$
(Contd.)
High performance liquid

Mobile phase

Chromatographic gradient

\begin{tabular}{llll}
\hline Time (min) & A & B & $\begin{array}{l}\text { Flow velocity } \\
(\mathrm{nL} \mathrm{min}\end{array}$ \\
\hline 0 & $95 \%$ & $5 \%$ & 600 \\
13 & $92 \%$ & $8 \%$ & 600 \\
99 & $78 \%$ & $22 \%$ & 600 \\
120 & $68 \%$ & $32 \%$ & 600 \\
122 & $10 \%$ & $90 \%$ & 600 \\
130 & $10 \%$ & $90 \%$ & 600 \\
$* 130$ min chromatographic gradient. & & \\
\hline
\end{tabular}

Mass spectrometer

Spray voltage

Capillary temperature

S-lens RF level

Collision energy

Isolation window

Resolution setting

Parent ion scan range

Data-dependent MS/MS
Thermo Scientific Easy-nLC 1000

A: $0.1 \%$ formic acid in water B: $0.1 \%$ formic acid in acetonitrile 130 min chromatographic gradient*
Thermo Scientific Orbitrap Fusion Lumos

\author{
$2.0 \mathrm{kV}$ \\ $275^{\circ} \mathrm{C}$ \\ 30 \\ $27 \% \mathrm{HCD}$ \\ $1.6 \mathrm{~m} / \mathrm{z}$ \\ Level one: 120000 @ m/z 200 \\ Level two: 30000 @ $\mathrm{m} / \mathrm{z} 200$ \\ $\mathrm{m} / \mathrm{z} 300-1500$ \\ Start from $m / z 110$ \\ Top-speed scan mode, $3 \mathrm{~s}$
}

chromatograph

Child ion scan range

The collected raw results were searched using the Proteome Discoverer 2.1 software; the search engine used Sequest HT. The database was downloaded using the uniprot_muscomorph.fasta database downloaded from the Uniprot website (Taxonomy ID: 43733, http://www.uniprot.org/), and the search results were filtered by $1 \%$ FDR at both peptide and protein levels. The search parameters are shown in the table below. The relative quantitative analysis of the identified proteins was also carried out in this experiment. The relative content is expressed by the emPAI value. ${ }^{18}$ This algorithm performs the theoretical enzymatic hydrolysis of each protein, and then calculates the ratio of the identified peptides to theoretical enzymatic peptides. The higher the number of identified peptides and the ratio, the higher the emPAI value. Gene ontology (GO) analyses were performed on the identified proteins using Proteome Discoverer's own Protein Center software.

\begin{tabular}{ll}
\hline Item & Value \\
\hline Enzyme & Trypsin \\
Max missed cleavages & 2 \\
Fixed modifications & Carbamidomethyl (C) \\
Variable modifications & Oxidation (M), acetyl (protein N-terminus) \\
Peptide mass tolerance & $\pm 10 \mathrm{ppm}$ \\
Fragment mass tolerance & $0.02 \mathrm{Da}$ \\
Peptide confidence & High \\
Peptide length & $\geq 6$ \\
Database & Uniprot-muscomorpha.fasta
\end{tabular}




\subsection{Animals and drug treatment}

Forty 2 month-old APPswe/PS1dE9 mice were purchased from Nanjing Biomedical Research Institute of Nanjing University. APPswe means that Lys and Met of 595596 loci of APP terminal sequence were replaced by Asn and Leu, respectively. PS1dE9 is the deletion mutation of the 9th exon dE9 of presenilin 1 gene. The two variations were all found in familial AD. The APPswe/ PS1dE9 mice are double transgenic positive mice, a recognized animal model of $\mathrm{AD},{ }^{16}$ which develop $\mathrm{AD}$ at about 6 to 7 months of age. ${ }^{19}$ These mice were randomly divided into four groups, including the model control group, the HL group, the Donepezil group (clinical positive control) and the proanthocyanidins group (natural antioxidant control), with 10 mice in each group. Ten 2 month-old C57BL/6 mice were obtained from the Guangdong Medical Laboratory Animal Center as the normal control group. All the animals were raised under specific pathogen-free conditions. All animal treatments were strictly in accordance with the National Institutes of Health Guide for the Care and Use of Laboratory Animals and were approved by the Institutional Ethical Committee (IEC) of Guangdong Pharmaceutical University. $0.25 \mathrm{~mL}$ of the drugs (HL: $400 \mathrm{mg} \mathrm{kg}{ }^{-1}$, Donepezil: $5.7 \mathrm{mg} \mathrm{kg}^{-1}$, and proanthocyanidins: $400 \mathrm{mg} \mathrm{kg}^{-1}$ ) were administered intragastrically for 180 days and the other two groups were given the same volume of saline.

\subsection{Morris water maze test}

To evaluate learning and memory, the Morris water maze test was performed for six days using the described method. ${ }^{20}$ The test was carried out in a circular pool without light and recorded by an automatic video analysis system. Water was poured into a circular tank and the temperature was kept at $25{ }^{\circ} \mathrm{C}$. Each mouse was allowed to find the platform for $90 \mathrm{~s}$ and stay on the platform for $15 \mathrm{~s}$. If the mouse did not find the platform, it was guided to the platform and allowed to stay on the platform for $15 \mathrm{~s}$. This training period lasted for five consecutive days and the platform position remained unchanged. On the sixth day, the platform was removed, and all the mice were put into the water from the same location. Each mouse swam freely for 90 s. During the whole experiment, the swimming track and the number of times of crossing the platform were recorded by a software.

\subsection{Step-down test}

A step-down test was performed after the Morris water maze test to measure the retention of memory according to the method described. ${ }^{21}$ Each mouse was placed on the platform after three minutes of adaptation to the environment of the step-down instrument. The power of the instrument was turned on and the test was started. The latency period (stepping down from the platform for the first time) and the number of errors (frequency of jumping off the platform) within 10 minutes were recorded. The test was repeated after 24 hours of training.

\subsection{Immunofluorescent staining}

The mice were deeply anaesthetized with $5 \%$ chloral hydrate, and then infused with $0.9 \%$ normal saline and $4 \%$ paraformaldehyde (PFA). Brain tissues were fixed with 4\% PFA for $24 \mathrm{~h}$, dehydrated with graded ethanol and xylene, embedded in paraffin and sliced into successive coronary sections $(5 \mu \mathrm{m})$. Sections were incubated with $\mathrm{A} \beta$ antibody $(1: 200)$ at $4{ }^{\circ} \mathrm{C}$ overnight. On the second day, sections were incubated with goat anti-rabbit IgG antibody (Alexa Fluor 488, 1 : 400) for 1 hour and 4,6-diamidino-2-phenylindole (DAPI) for 5 minutes under $37{ }^{\circ} \mathrm{C}$ in dark. Images were obtained with a fluorescence microscope and analyzed with Image J.

\subsection{Data analysis}

The data were analyzed with the Prism 5 software and expressed as mean \pm standard deviation (SD). Statistical comparisons between groups were evaluated by $t$-test with the Prism 5 software. $P$ values less than 0.05 were considered statistically significant.

\section{Results}

\subsection{GO analysis of the total composition of protein from the HL powder}

A range of proteins have been identified via the LC-MS/MS analysis. Fig. 1A displays the histogram of the cellular component associated with proteins identified in the HL powder. The proportions of cell membrane, cytoplasm and ribosome were $32.49 \%, 20.14 \%$ and $13.96 \%$, respectively. The pie graph of the biological processes is shown in Fig. 1B, among which the metabolic process accounted for $54.43 \%$, while transport, regulation of biological processes, response to stimulus, cell organization and biogenesis, and other accounted for $14.43 \%$, $12.95 \%, 7.50 \%, 6.25 \%$, and $4.43 \%$, respectively. The pie graph of the molecular functions is shown in Fig. 1C. Notably, the catalytic activity accounted for the highest proportion of the molecular function, which was $35.96 \%$. Nucleic acid binding, protein binding and iron binding come in second, with a total proportion of $39.84 \%$. GO analysis revealed that the identified proteins were predominantly catalytic proteins and binding proteins, which were mainly located in the membrane and cytoplasm, and involved in various metabolic processes, transport and regulation of biological processes.

\subsection{Details of the target protein from the HL powder}

More than 2000 highly reliable proteins were found in the study. A list of the 8 meaningful proteins identified in the HL powder are shown in Table 1. Among them, some antioxidant proteins have a relatively high content, such as the AhpC/TSA family, catalase and superoxide dismutase. In addition, the relative quantitative analysis of the identified proteins was carried out in this experiment. Finally, we have provided a list of peptide sequences within the proteins identified in the HL powder (Table 2) and the MS2 spectra of annotated characteristic peptides (Fig. 2). According to the MS2 spectra of these proteins, most of the protein sequence matching degree reached $60 \%$, and the identified $b, y$ fragment ions were continuous, which means that the identification results were highly reliable. 
A

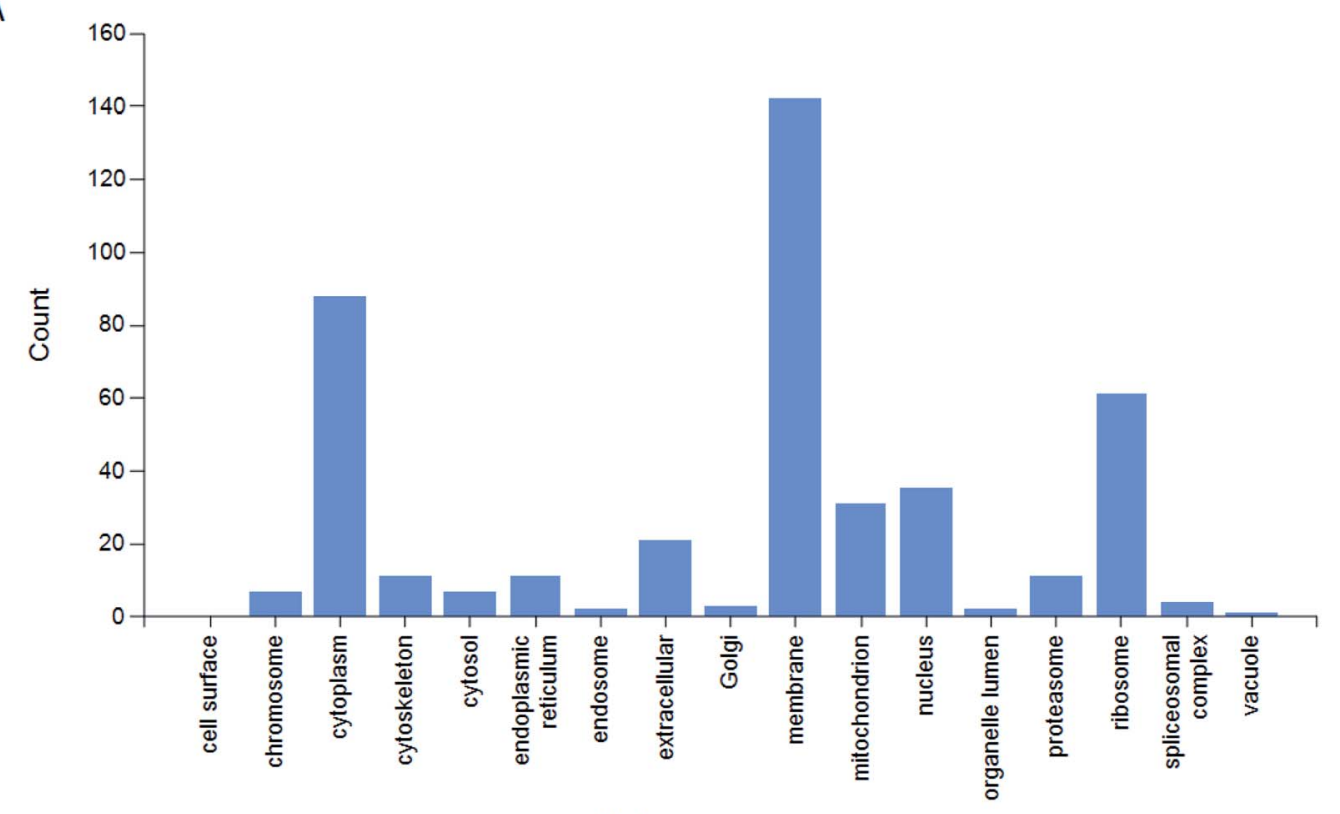

B

cellular component
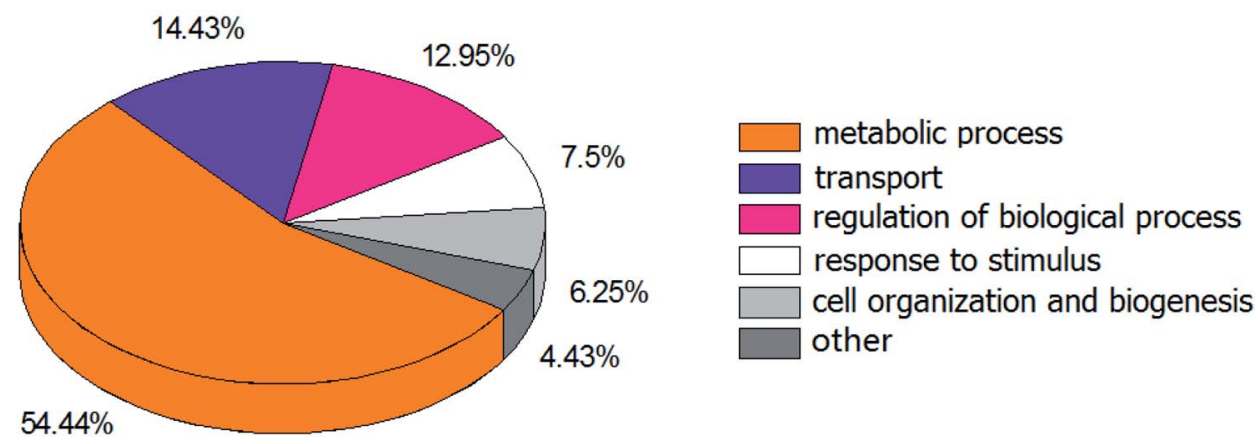

biological process

C
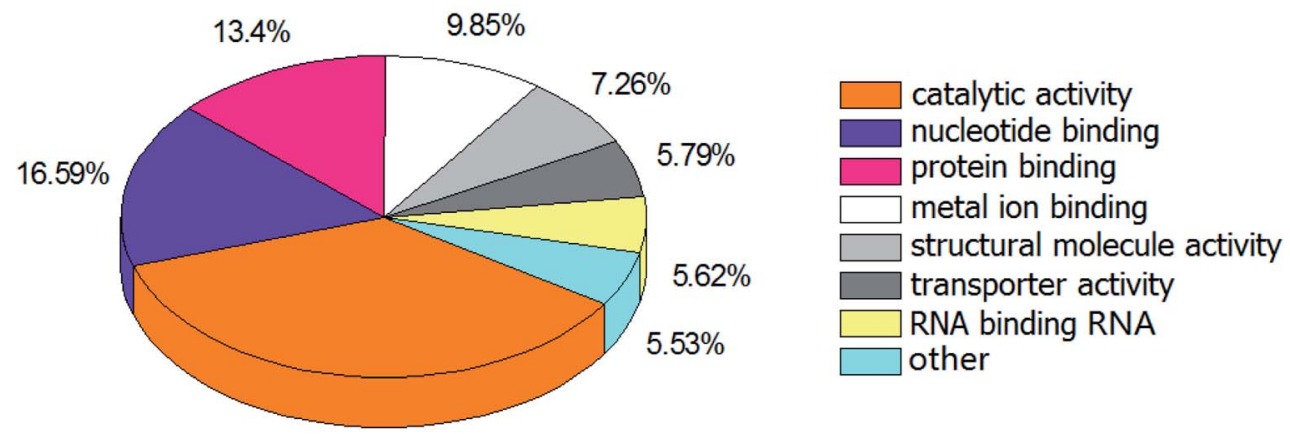

$35.96 \%$

molecular function

Fig. $1 \mathrm{GO}$ analysis related to identified proteins from the housefly larvae powder. (A) Cellular component, (B) biological process and (C) molecular function.

3.3. Preliminary biological effects of the HL powder on Alzheimer's transgenic mice

3.3.1 HL powder treatment simplifies the track and reduces the number of times of crossing the platform of
Alzheimer's mice in the Morris water maze test. Mice in the normal control group had a simple movement track and hardly left the platform quadrant. Mice in the model control group had complex movement trajectories that spanned four quadrants, 
Table 1 List of proteins (accession number, protein name, coverage, MW, emPAl, score) identified in the HL powder

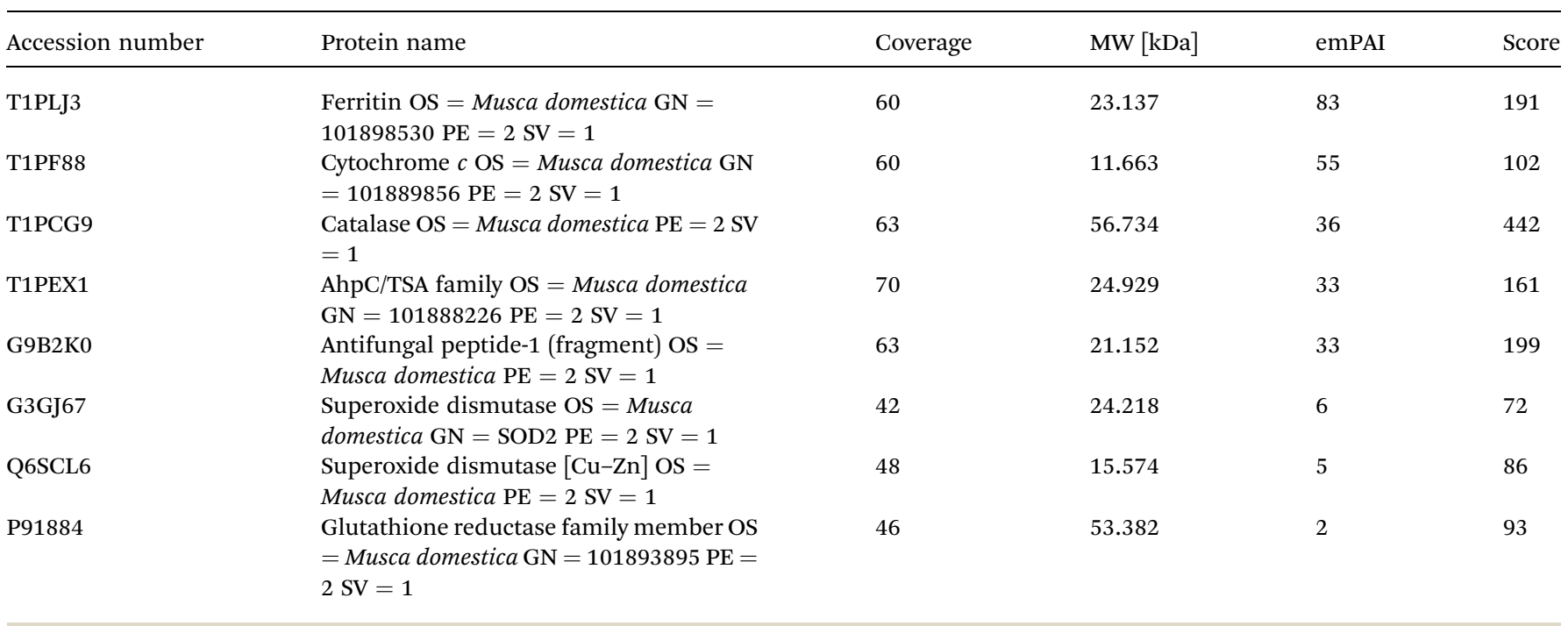

and finally did not return to the quadrants where the platform was located. Compared with the model control group, the movement tracks of the Donepezil group, the HL group and the proanthocyanidins group were simpler. Compared with the Donepezil group and the proanthocyanidins group, the $\mathrm{HL}$ group has significantly shorter trajectories and significantly fewer quadrants (Fig. 3A). Compared with the control group, the number of times of crossing the platforms of the model control group, the Donepezil group, the HL group and the proanthocyanidins group was significantly reduced $(P<0.05)$. Compared with the model control group, the number of times of crossing platforms $(P<0.05)$ of the HL group significantly increased, while that of the Donepezil group and the proanthocyanidins group were not significantly different $(P>0.05$, Fig. 3B).

3.3.2 HL powder treatment reduces the latency period and the number of errors in the step-down test. As shown in Fig. $4 \mathrm{~A}$ and $\mathrm{B}$, compared with the normal control group, the latency period of the model control group was significantly shortened, and the number of errors significantly increased on the first day of training $(P<0.05)$. Compared with the model group, the

Table 2 List of peptide sequences found within the protein sequences identified in the HL powder

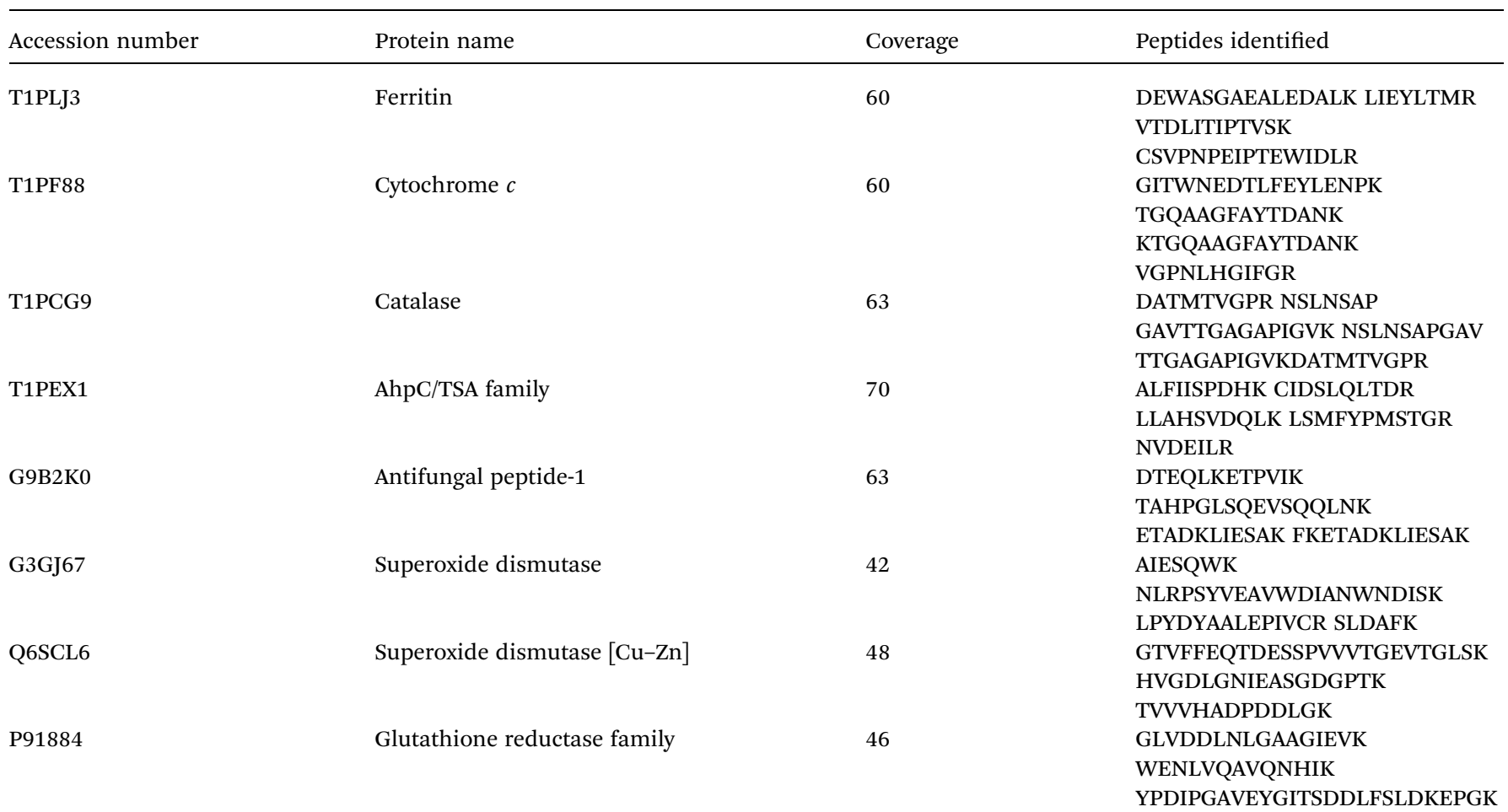


A

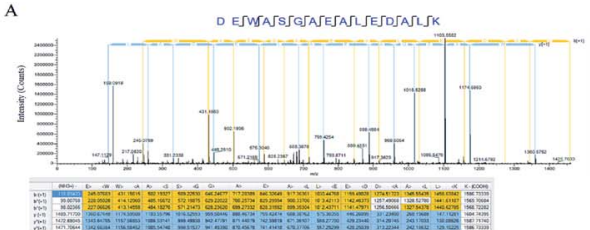

$\mathrm{C}$

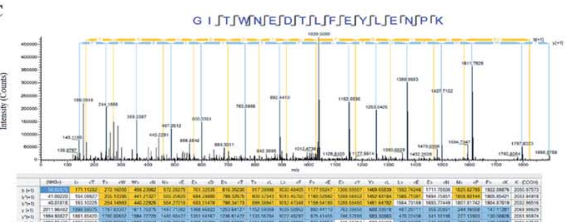

$\mathrm{E}$
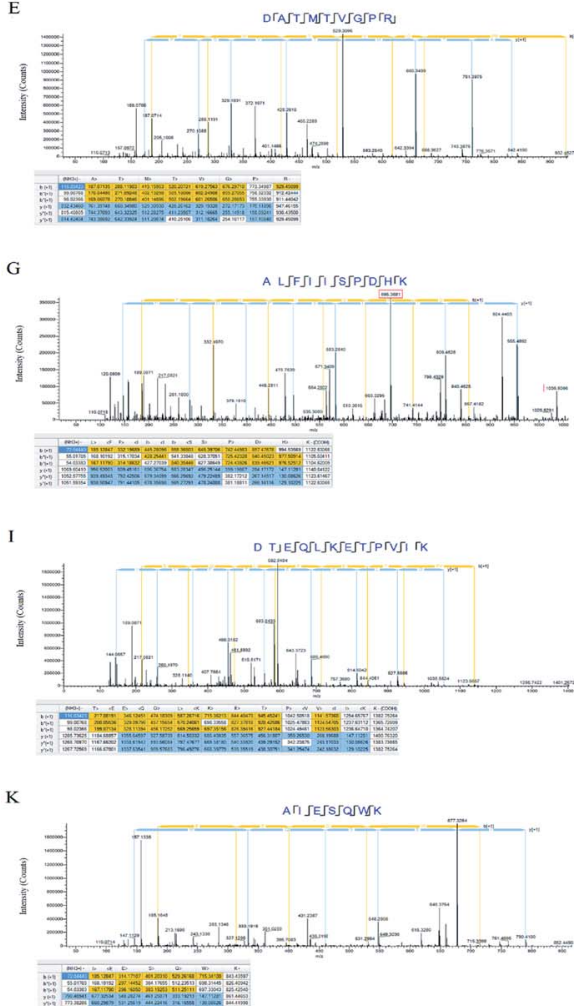

M
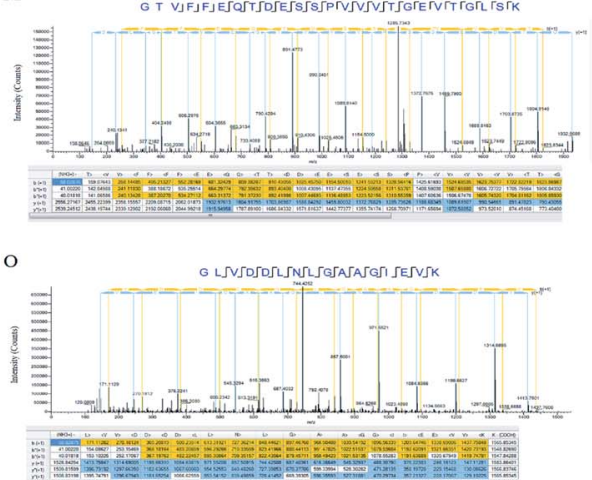

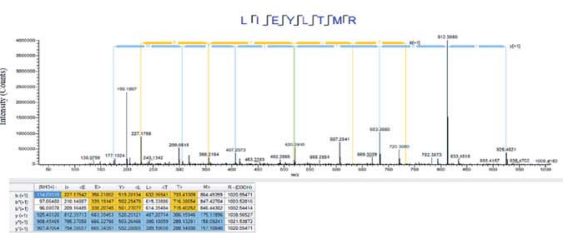

D

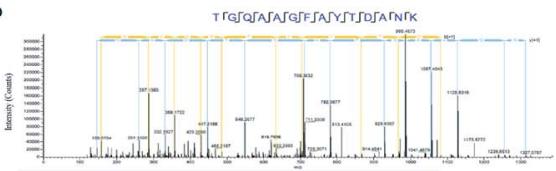

mas a

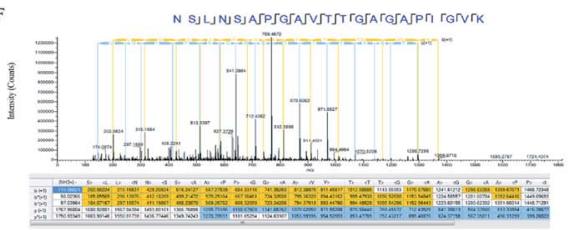

H
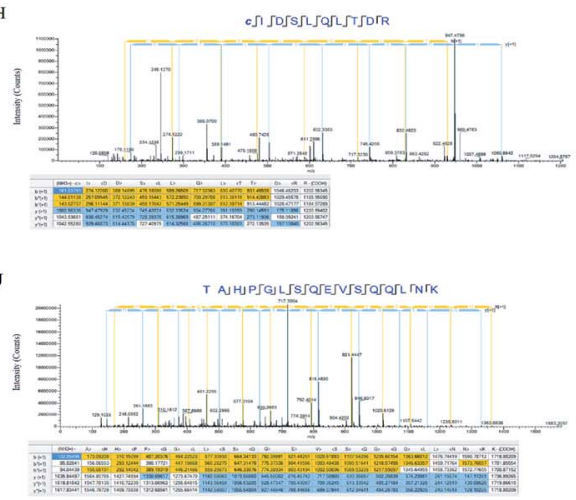

L

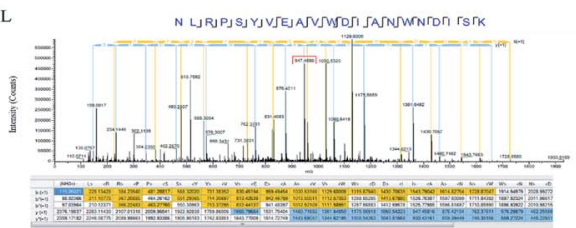

$\mathrm{N}$
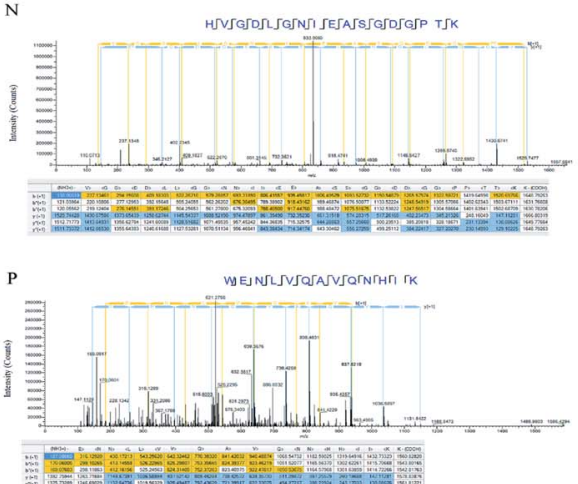

Fig. 2 MS2 spectrum of annotated characteristic peptides found within the protein sequences identified in HL powder. (A and B) Ferritin. (C and D) Cytochrome c. (E and F) Catalase. (G and H) AhpC/TSA family. (I and J) Antifungal peptide-1. (K and L) Superoxide dismutase. (M and N) Superoxide dismutase [Cu-Zn]. (O and P) Glutathione reductase family member. 
A

$\mathrm{NC}$

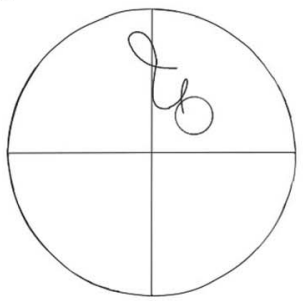

$\mathrm{HL}$

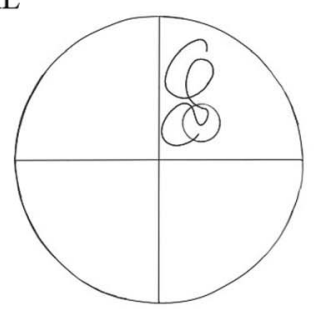

MC

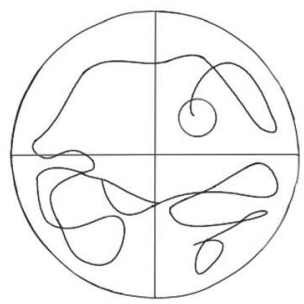

PC

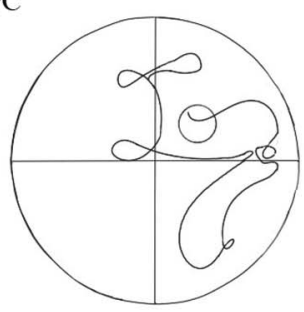

Donepezil

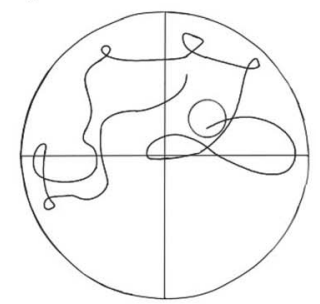

B

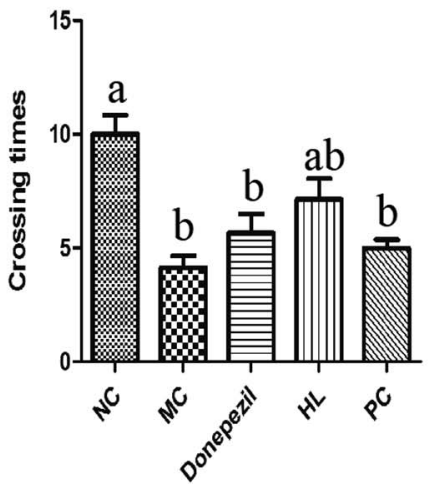

Fig. 3 Experimental results of the Morris water maze in each group. (A) Representative track map of finding platform on the fifth day of water maze training for each group of mice. (B) The number of times each group of mice crossed the platform on the sixth day (NC: the normal control group; MC: the model control group; Donepezil: the Donepezil group; HL: the HL group; PC: the proanthocyanidins group; a: $P<0.05$ vs. MC; $b$ : $P<0.05$ vs. NC).

latency periods of the other groups were not significantly different as well as the number of errors $(P>0.05)$. The next day, compared with the normal control group, the latency periods of the model control group, the Donepezil group and the proanthocyanidins group were significantly shortened $(P<$ $0.05)$, and the number of errors increased significantly $(P<$ 0.05), while there was no significant difference between the HL group and the normal control group $(P>0.05)$. Compared with
A

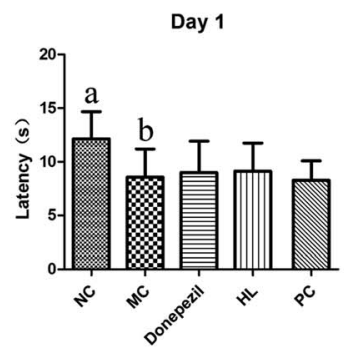

B

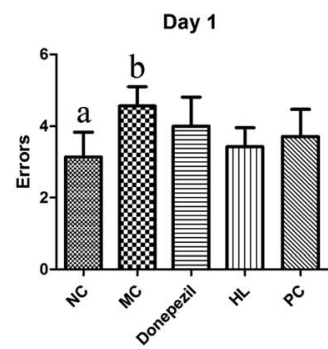

$\mathrm{C}$

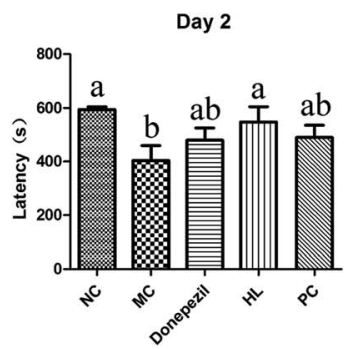

D

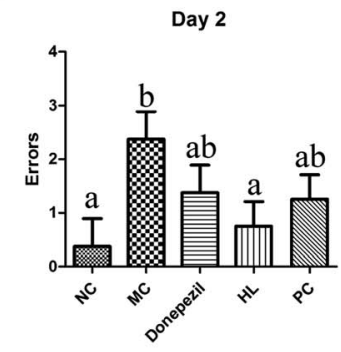

Fig. 4 Experimental results of the step-down test in each group. (A) Latency period in each group on day 1. (B) Number of errors in each group on day 1. (C) Latency period in each group on day 2. (D) Number of errors in each group on day 2 (NC: the normal control group; MC: the model control group; Donepezil: the Donepezil group; HL: the HL group; PC: the proanthocyanidins group; $a$ : $P<0.05$ vs. $M C$; b: $P<0.05$ vs. NC). 
A

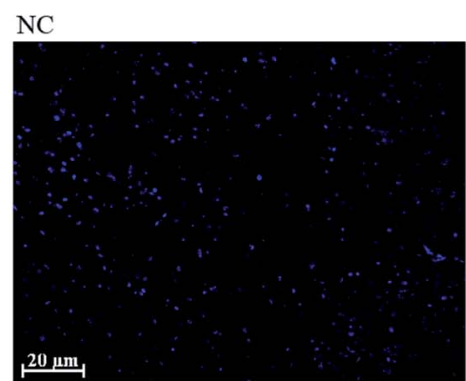

$\mathrm{HL}$

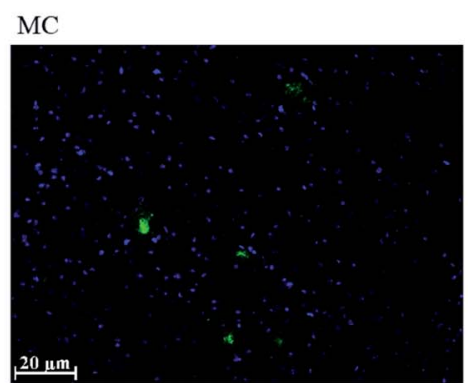

PC
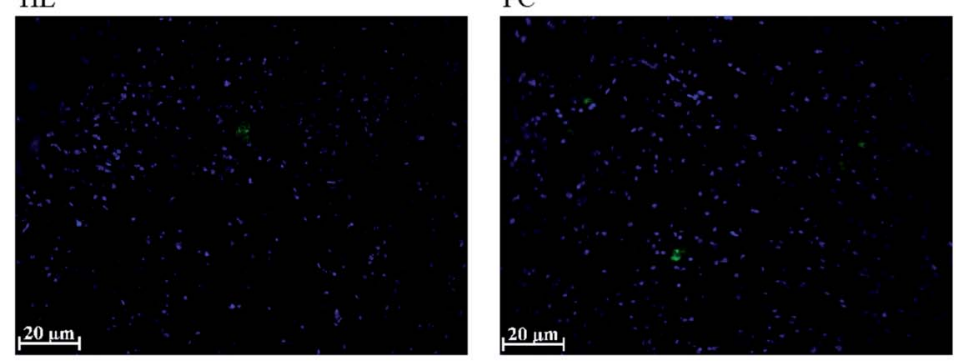

Donepezil

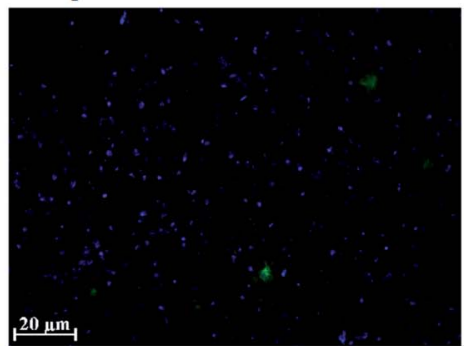

B

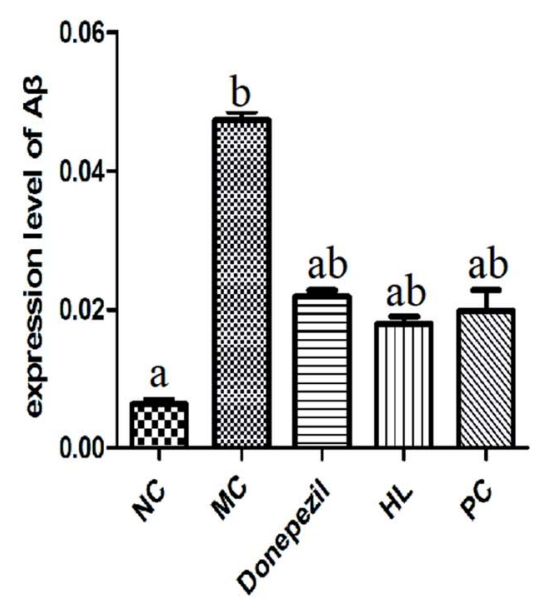

Fig. 5 Immunofluorescent staining of $A \beta$ deposition in cerebral cortex regions of mice in each group. (A) Photomicrograph of $A \beta$ deposition in each group (scale bar $=20 \mu \mathrm{m}$ ). (B) The analyzed data of $A \beta$ deposition in each group by Image J. Data are presented as mean \pm SEM (NC: the normal control group; MC: the model control group; Donepezil: the Donepezil group; HL: the HL group; PC: the proanthocyanidins group; a: $P<$ 0.05 vs. MC; b: $P<0.05$ vs. NC).

the model control group, the latency periods of the Donepezil group, the HL group and the proanthocyanidins group were significantly prolonged $(P<0.05)$, and the number of errors was significantly reduced $(P<0.05$, Fig. $4 \mathrm{C}$ and $\mathrm{D})$.

3.3.3 HL powder treatment reduces $A \beta$ deposition in the cerebral cortex of Alzheimer's mice. In order to further verify the protective effect of the HL powder on APP/PS1 mice, immunofluorescent stain was used to detect the $\mathrm{A} \beta$ deposition in cerebral cortex regions. As shown in Fig. 5, there was no $\mathrm{A} \beta$ deposition in the cortical region of the normal control group, while there was significant $A \beta$ deposition in the cortical region of the model control group. The optical density statistics showed that compared with the normal control group, the fluorescence markers in the cortical areas of the model control group, the Donepezil group, the HL group and the proanthocyanidins group were significantly enhanced $(P<0.05)$. Compared with the model control group, the fluorescence labeling intensities of the cortical areas in the Donepezil group, the HL group and the proanthocyanidins group were significantly reduced $(P<0.05)$.

\section{Discussion}

$\mathrm{HL}$ is an excellent source of high-quality protein, including polyunsaturated fats, vitamins, minerals and other nutrients for humans and animals. ${ }^{22}$ The crude HL extract has many pharmacological effects, such as anti-tumor, antibacterial, hypoglycemic, and hypolipidemic. However, the specific 
pharmacological material basis is not clear. In this study, HL was ground into powder for protein extraction and enzymatic hydrolysis, and the proteins were identified by LC-MS/MS. Then, the classical data-dependent acquisition technique was used. ${ }^{23}$ The initial result of the test was peptide information. Each protein has its own unique peptide. Only when the unique peptide of a certain protein was identified did we consider the protein to be identified. This method is currently recognized as the mainstream proteomics identification technology. In order to ensure the reliability of the results, we adopted false discovery rate (FDR) less than or equal to $1 \%$ at the peptide level and $Q$ value less than or equal to $1 \%$ at the protein level as the screening conditions. Finally, we identified more than 2000 highly reliable proteins and conducted a relative quantitative analysis for each identified protein. The results showed that HL contain a series of meaningful proteins, such as catalytic related proteins, antioxidant proteins and antimicrobial peptides. The most important proteins with high contents are ferritin, cytochrome $c$, AhpC/TSA family, catalase, superoxide dismutase, and antifungal peptide- 1 .

Iron is an essential nutrient for almost all organisms. It is a cofactor for many cell life activities and plays a key role in cell growth. ${ }^{24}$ However, an excess of free iron produces ROS and hydroxyl radicals through the Fenton reaction. ${ }^{25}$ The most important biological functions of ferritin are balancing iron metabolism and storing iron in a bioavailable and non-toxic form. Ferritin can remove excess iron to protect cells from oxidative stress. ${ }^{26}$ In this way, ferritin can maintain the stability of iron pool, balance the iron level in cells and organisms, and decrease oxidative damage caused by iron toxicity. ${ }^{27}$ Moreover, ferritin can inhibit the utilization of iron by invading pathogenic microorganisms, and therefore, can participate in immune responses to pathogen infection. ${ }^{\mathbf{2 8 , 2 9}}$ Qin et al. showed that the housefly protein powder can effectively enhance the humoral immune function in normal mice and has certain preventive and therapeutic effects on the immune function impairment caused by cyclophosphamide. ${ }^{30}$ Ai et al. suggested that the protein-enriched fraction from HL might possess the ability to activate NK cells (natural killer cells) to improve the immune function of the body. ${ }^{31}$ Sun et al. indicated that the peptide fraction from HL has an immunomodulatory activity and it's in vivo anti-tumor activity might be achieved by activating Th1-based protective cell-mediated immunity. ${ }^{32}$ Although the immune regulation mechanism of HL has been discussed with regards to different aspects, no one has explored it from the aspects of iron metabolism and ferritin. The immune regulation mechanism of HL can be further explored from the perspective of iron metabolism and ferritin, which may provide a new perspective for future relative research.

Cytochrome $c$ is a kind of protein containing heme, which is an electron transporter in the process of biological oxidation. In normal physiological conditions, $98 \%$ of the mitochondria oxygen consumption is used to make ATP, and the remaining $2 \%$ is used to produce hydrogen peroxide $\left(\mathrm{H}_{2} \mathrm{O}_{2}\right)$. However, in pathological conditions, the $\mathrm{H}_{2} \mathrm{O}_{2}$ oxygen consumption will greatly exceed the level of $2 \%$. The cytochrome $c$ in the mitochondria can remove superoxide radicals $\left(\mathrm{O}_{2}{ }^{-}\right)$and $\mathrm{H}_{2} \mathrm{O}_{2}$ through electronic by-pass leakage. ${ }^{33,34}$ The AhpC/TSA family has an extensive intracellular reductive activity. Alkyl hydrogen peroxide reductase (AhpC) can oxidize intracellular $\mathrm{H}_{2} \mathrm{O}_{2}$, alkyl hydrogen peroxide and organic peroxide into corresponding alcohols, and also has the ability to reduce intracellular peroxynitrite. ${ }^{35,36}$ Thiol-specific antioxidants (TSA) are an important component of the enzyme defense system against sulfurcontaining free radicals. Therefore, the AhpC/TSA family is a group of highly active antioxidant proteins. Catalase (CAT) is an anti-inflammatory enzyme that converts $\mathrm{H}_{2} \mathrm{O}_{2}$ into $\mathrm{H}_{2} \mathrm{O}$ and oxygen. ${ }^{37}$ Superoxide dismutase (SOD) is an enzyme that catalyzes the decomposition of the $\mathrm{O}_{2}{ }^{-}$, and is the main antioxidant enzyme in the scavenging mechanism; it can also prevent the reaction of $\mathrm{H}_{2} \mathrm{O}_{2}$ and $\mathrm{O}_{2}{ }^{-}-$that produces hydroxyl radical $\left(\mathrm{OH}^{\circ}\right)$, which causes a greater damage to the organism. The activity of antioxidant enzymes reflects the ability of cells to remove ROS and resist oxidative damages. The changes in the activity of antioxidant enzymes will disrupt the balance between the intracellular oxidation and antioxidant system, leading to the excessive accumulation of reactive oxygen species (ROS), oxidation of membrane phospholipids, and ultimately to apoptosis. ${ }^{38}$ Zhang et al. indicated that HL protein hydrolysates display high antioxidant activities and free radical scavenging activities. ${ }^{39}$ Li et al. showed that feeding bull frogs with the HL powder increased their total antioxidant capacity, and that the activity of SOD and CAT increased linearly with the increase of the HL powder inclusion level. ${ }^{40} \mathrm{Ai}$ et al. proved that the proteinenriched fraction from HL has antioxidant and free radical scavenging effects, which can not only significantly increase the activity of SOD and glutathione peroxidase (GSH-Px), but also depress the production of malondialdehyde (MDA) in liver homogenate of mice through autooxidation and inhibit the hepatic mitochondrial expansion induced by the $\mathrm{Fe}^{2+}$-ascorbic acid system. ${ }^{31}$ He et al. demonstrated that the HL powder achieves antioxidant effects by inducing the increased expression of SOD and CAT in the brain. ${ }^{16}$ Currently, there have been a lot of studies on the antioxidant effects of HL, and some mechanistic studies have been carried out, but none of them have explored the mechanism from the perspective of these antioxidant proteins contained in HL itself. The protein identified above may be the material basis for the crude HL extract to exert an antioxidant effect.

In recent years, the incidence of fungal infections has increased year by year due to the abuse of broad-spectrum antimicrobial agents. Antifungal peptides are small molecular peptides for inhibiting or killing fungi. The antifungal peptides have high antimicrobial activity and stability. The mechanism of antimicrobial peptides is to attack bacteria by associating with the bacterial membrane and forming transmembrane pores that facilitate the unrestricted transport of ions, which can be applied to drug-resistant bacterial strains. ${ }^{\mathbf{4 1}}$ The development of antifungal peptides provides a new way to solve the problem of fungal resistance. ${ }^{42}$ Studies on the antifungal activity of HL extracts have increased year by year, wherein they isolated and purified antifungal peptides from the extract, and carried out a series of experiments. Zhou et al. revealed that the antifungal peptide-1A from HL has excellent antiviral activities 
and antifungal activities. ${ }^{\mathbf{4 3}}$ The antifungal peptide- 1 identified in this study may be one of the material basis for HL to exert its antibacterial effect. In order to carry out further in-depth research, we published its peptide sequences (Table 2). In addition, we have obtained quite a few other proteins, which should not be neglected because they may make important contributions to the pharmacological effects of HL.

$\mathrm{AD}$ is a degenerative disease of the central nervous system, mainly manifested as progressive memory disorder, cognitive dysfunction and other neuropsychiatric symptoms. The extracellular senile plaques formed by amyloid beta $(\mathrm{A} \beta)$ deposition is the main characteristic pathological change of AD. Since oxidative stress is one of the important pathogenesis of $\mathrm{AD}$, and since the HL powder is rich in many antioxidant proteins, we further investigated the possible protective effect of the $\mathrm{HL}$ powder on Alzheimer's transgenic mice.

The results showed that the HL powder significantly improved the memory ability of Alzheimer's mice, and reduced the deposition of $\mathrm{A} \beta$. It was even better than the commonly used clinical drug Donepezil and natural antioxidant proanthocyanidins to some extent. We have previously reported that the HL powder protects against memory damage in Alzheimer's transgenic mice through regulating JNK and P38 MAPK signaling pathways and regulating UCP4 and CyclinD1 expression. ${ }^{\mathbf{1 6}}$ However, after the comprehensive identification of the $\mathrm{HL}$ powder, we found that the protective mechanism explored was limited. It may also be related to lipoprotein and ferritin metabolism. We speculate that the HL powder might play a protective role on $\mathrm{AD}$ from multiple perspectives. Of course, we also believe that HL has an important application value not only in $\mathrm{AD}$, but also in other medical diseases and health care.

In this study, we identified some antioxidant proteins from the HL powder, and preliminarily verified that HL powder has a protective effect on $\mathrm{AD}$. However, we did not explore which proteins play a protective role, or whether several of them combine to play this role. In the following studies, we will conduct further research of these identified proteins.

\section{Conclusion}

In conclusion, we identified the protein expression profiles and obtained the amino acid sequences of these proteins of HL in this study, which provided a material basis for further medical study of HL. We hope that the results of this work will give new ideas for the researchers in further exploring the physiological functions of these identified proteins and clarifying the specific pharmacological material basis of the medical effect of HL.

\section{Conflicts of interest}

On behalf of all the authors, I declare that we have no conflicts of interest.

\section{Acknowledgements}

The present study was supported by grants from the National Natural Science Foundation of China (81401150, 81703977).

\section{References}

1 R. W. Pemberton, J. Ethnopharmacol., 1999, 65, 207-216.

2 R. Lin, H. X. Bian, C. Guo, Y. Y. Fan, Y. Guan and M. M. Xi, China Pharm., 2017, 28, 558-561.

3 R. A. Sherman, Evid. Based Complement. Altern. Med., 2014, 113.

4 G. Bai and Y. X. Zhang, J. Baotou Med. Coll., 2010, 26, 10-13. 5 J. Niu, X. X. Cheng, Q. Yang, Z. P. Chen, H. X. Jun, X. M. Tang, R. H. Zheng, L. M. Pan and C. R. Zhang, Mod. Chin. Med., 2016, 18, 1371-1375.

6 S. O. Park, J. H. Shin, W. K. Choi, B. S. Park, J. S. Oh and A. Jang, J. Environ. Biol., 2010, 31, 865-871.

7 F. J. Chu, X. B. Jin and J. Y. Zhu, J. Atheroscler. Thromb., 2011, 18, 282-290.

8 F. J. Chu, X. B. Jin, Y. Y. Xu, Y. Ma, X. B. Li, X. M. Lu, W. B. Liu and J. Y. Zhu, Evid. Based Complement. Alternat. Med., 2013, 1-10.

9 L. Zhu, P. Wang, Q. L. Qin, H. Zhang and Y. J. Wu, Food Chem. Toxicol., 2013, 60, 385-390.

10 L. X. Hou, Y. H. Shi, P. Zhai and G. W. Le, J. Ethnopharmacol., 2007, 111, 227-231.

11 K. Suresh Kumar, K. Kamatam and W. Hui-Fen, Anal. Chem., 2008, 80, 9681-9688.

12 S. K. Kailasa and H. F. Wu, Talanta, 2010, 83, 527-534.

13 S. K. Kailasa, V. N. Mehta and H. F. Wu, $R S C A d v .$, 2014, 4, 16188-16205.

14 S. K. Kailasa, N. Hasan and H. F. Wu, Talanta, 2012, 97, 539549.

15 Y. Wang, J. F. Xiu, J. Z. Cheng, P. Zhao, X. L. Shang, T. Wang and J. W. Wu, Biomed. Environ. Sci., 2016, 29, 56-65.

16 Y. R. He, X. Yang, M. Y. Jiao, A.-D. Shailendra, Y. Zeng and H. F. Mei, Food Funct., 2019, 10, 235-243.

17 H. F. Mei, J. H. Xu, Y. R. He, X. Yang, W. B. Liu, W. Tian, Y. Zeng and J. Y. Zhu, J. Biosci., 2018, 43, 969-983.

18 M. A. Bilen, T. Pan, Y.-C. Lee, S.-C. Lin, G. Yu, J. Pan, D. Hawke, B.-F. Pan, J. Vykoukal and K. Gray, J. Proteome Res., 2017, 16, 2709-2728.

19 R. S. Reiserer, F. E. Harrison, D. C. Syverud and M. P. Mcdonald, Genes, Brain Behav., 2007, 6, 54-65.

20 R. H. Lin, L. Li, Y. Z. Zhang, S. Huang, S. J. Chen, J. Shi, P. Y. Zhuo, H. Jin, Z. F. Li and W. L. Liu, Biol. Res., 2018, 51, 21.

21 Y. M. Kim, E. S. Ji, S. H. Kim, T. W. Kim, I. G. Ko, J. J. Jin, C. J. Kim, T. W. Kim and D. H. Kim, Journal of Exercise Rehabilitation, 2015, 11, 5-11.

22 G. D. Ren and A. M. Shi, Entomol. Knowl., 2002, 17, 1-5.

23 J. P. Koelmel, N. M. Kroeger, E. L. Gill, C. Z. Ulmer, J. A. Bowden, R. E. Patterson, R. A. Yost and T. J. Garrett, J. Am. Soc. Mass Spectrom., 2017, 28, 908-917.

24 N. C. Andrews, Blood, 2008, 112, 219-230.

25 N. C. Andrews and P. J. Schmidt, Annu. Rev. Physiol., 2007, 69, 69-85.

26 K. Orino and W. Kiyotaka, Vet. J., 2008, 178, 191-201. 27 F. M. Torti and S. V. Torti, Blood, 2002, 99, 3505-3516. 
28 G. Beck, W. E. Thomas, S. H. Gail, F. S. Samuel and J. M. John, Dev. Comp. Immunol., 2002, 26, 11-26.

29 D. S. T. Ong, W. Lihui, Z. Yong, B. Ho and D. Jeak Ling, J. Endotoxin Res., 2005, 11, 267-280.

30 R. G. Qin, F. U. Ping, J. W. Wu and G. Guo, J. Chin. Med. Mater., 2007, 30, 635-637.

31 H. Ai, F. R. Wang, N. Zhang, L. Y. Zhang and C. L. Lei, J. Insect Sci., 2014, 13, 112.

32 H. X. Sun, L. Q. Chen, J. Zhang and F. Y. Chen, J. Ethnopharmacol., 2014, 153, 831-839.

33 Y. Zhao and, J. Biol. Chem., 2003, 278, 2356-2360.

34 Z. B. Wang, M. Li, Y. Zhao and J. X. Xu, Protein Pept. Lett., 2003, 10, 247-253.

35 J. A. Imlay, Curr. Opin. Microbiol., 2015, 24, 124-131.

36 S. Sankaralinga, K. Das and S. Dhandayuthapani, J. Bacteriol., 2015, 197, 51-62.
37 I. Ali, S. N. Khan, C. Chatzicharalampous, D. Bai and H. M. Abu-Soud, J. Inorg. Biochem., 2019, 197, 110706.

38 M. L. Hao and L. R. Tao, Spectrochim. Acta, Part A, 2019, 220, 117104.

39 H. Zhang, P. Wang, A. J. Zhang, X. Li, J. H. Zhang, Q. L. Qin and Y. J. Wu, Acta Biol. Hung., 2016, 67, 236-246.

40 X. Li, S. Rahimnejad, L. Wang, K. L. Lu, K. Song and C. X. Zhang, Aquaculture, 2019, 499, 295-305.

41 D. S. Bolintineanu, V. Vivcharuk and Y. N. Kaznessis, Int. J. Mol. Sci., 2012, 13, 11000-11011.

42 J. Cruz, C. Ortiz, F. Guzmán, R. Fernández-Lafuente and R. Torres, Curr. Med. Chem., 2014, 21, 2299-2321.

43 J. Zhou, L. Kong, N. Fang, B. Mao and H. Ai, J. Med. Entomol., 2016, 53, 1467-1472. 